\title{
Vaccins : spécificités, simulation de l'impact et modélisation médico-économique
}

\author{
Odile Launay ${ }^{1}$, Didier Hoch ${ }^{2}$, Christelle Saint-Sardos ${ }^{2}$, Didier Guillemot ${ }^{3}$ et les participants à la Table Ronde \\ $n^{\circ} 6$ de Giens $X X V^{\star}$ \\ 1 Université Paris Descartes, AP-HP, Inserm, Centre d'Investigation Clinique de Vaccinologie Cochin Pasteur (CIC BT505), Paris, France \\ 2 Sanofi Pasteur MSD, Lyon, France \\ 3 Unité de Pharmacoépidémiologie et Maladies Infectieuses Institut Pasteur/Inserm U 657, Paris, France
}

Texte reçu le 26 avril 2010 ; accepté le 15 juin 2010

\begin{abstract}
Mots clés :
vaccin ;

vaccinologie ;

recherche clinique ;

recherche

translationnelle ;

centre d'investigation

clinique ;

réseau ;

Résumé - Dans un contexte où plusieurs vaccinations ont été récemment recommandées en France, et où de nombreux nouveaux vaccins sont en développement, la Table Ronde a réalisé un état des lieux des spécificités du vaccin en France, que sont le développement clinique, la modélisation médico-économique, l'évaluation et la communication. Dans chacun de ces domaines ont été identifiés les étapes et processus clés à améliorer, assortis de propositions. Les principaux enjeux stratégiques sont de favoriser le développement des vaccins en France par l'accès à un suivi de populations caractérisées à large échelle, d'anticiper et coordonner l'évaluation des vaccins par les différents intervenants, d'accroître la communication autour des stratégies vaccinales, afin d'améliorer la perception des vaccins, l'acceptabilité des vaccinations, et d'augmenter la couverture vaccinale. La Table Ronde a également proposé la création d'un groupe de travail qui s'assurera de la poursuite, du suivi et de la mise en œuvre des propositions formulées.
\end{abstract}

épidémiologie ;

modélisation

économique

\section{Introduction}

La Table Ronde $n^{\circ} 6$ «Vaccins : spécificités, simulation de l'impact et modélisation médico-économique » s'est inscrite dans le contexte :

- de la recommandation récente de plusieurs vaccins en France : vaccins contre les infections à papillomavirus humain, ${ }^{[1,2]}$ pneumococcique conjugué, ${ }^{[3]}$ méningococcique conjugué, ${ }^{[4]}$

- et du développement actuel de nombreux nouveaux vaccins : environ une centaine de vaccins sont en cours de développement, parmi lesquels les vaccins contre les infections à : staphylocoque doré, méningocoque $\mathrm{B}$, chlamydiae, dengue, herpès, virus de l'immunodéficience humaine (VIH), hépatite $\mathrm{C}$, paludisme. ${ }^{[5]}$

En France comme dans le monde, la vaccination reste l'un des meilleurs outils de prévention des maladies infectieuses graves. Par ailleurs, le vaccin est un domaine thérapeutique d'ex-

\footnotetext{
* Pour la liste des participants, voir en fin d'article.
}

cellence pour la France : l'intérêt de santé publique, politique et industriel de cette excellence justifie que l'attractivité française soit entretenue.

L'objectif de la Table Ronde était de faire un état des lieux des spécificités des vaccins en identifiant les étapes et processus clés à améliorer tout au long de la vie des vaccins, et de proposer un plan d'action.

Le travail de la Table Ronde s'est organisé autour d'exposés, qui ont donné lieu à discussions et débats : «Les spécificités du vaccin » (D. Hoch), «Les spécificités du développement vaccinal, critères de choix pour la réalisation des essais d'enregistrement» (B. Fritzell), «Vaccination et impact épidémiologique » (P.-Y. Boelle), «Modélisation coût-efficacité » (B. Dervaux), «Place des modèles dans les recommandations vaccinales » (D. Levy-Brühl), «Sécurité, spécificités du vaccin » (J.-P. Fagot), «Perception et communication autour des vaccins » (D. Floret), « De l'AMM à la vaccination » (C. Saint-Sardos). Les débats se sont limités à la vaccination préventive. 


\section{Nombre de sujets enrolés dans les études cliniques de Phase III}

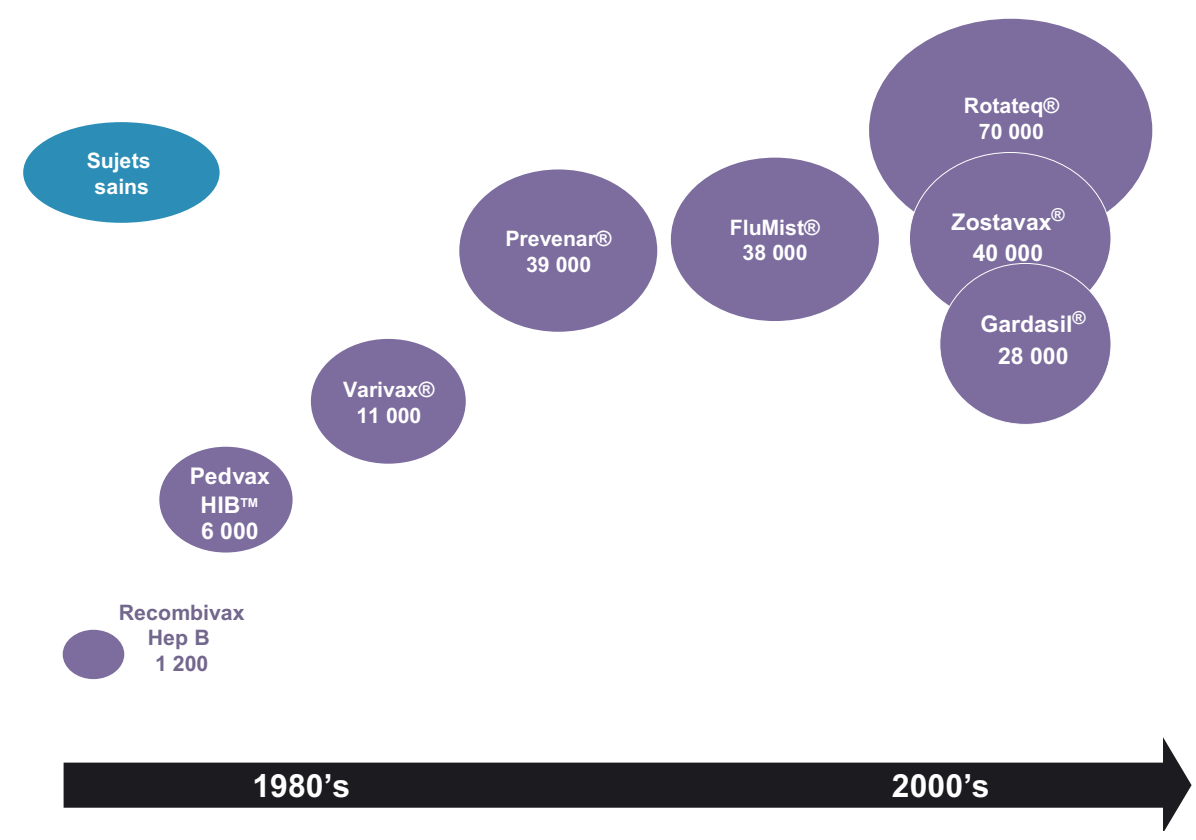

Fig. 1. Nombre de sujets enrôlés dans les études cliniques de développement vaccinal de phase III.

Cet état des lieux a permis de mettre en évidence les quatre grands domaines de spécificité du vaccin que sont : le développement clinique, la modélisation médico-économique, l'évaluation en termes de santé publique et de recommandation et la communication.

\section{Spécificités du vaccin en termes de développement clinique et de suivi}

\section{1. État des lieux}

Le vaccin est l'un des rares outils de santé publique médicamenteux utilisé en prévention primaire, et administré à des individus «sains » afin de diminuer un risque de survenue d'une maladie infectieuse en général et non à des personnes malades ou présentant des facteurs de risque, contrairement aux médicaments dits «classiques». Par nature, les populations cibles sont généralement de très larges populations définies sur des critères démographiques (par exemple, les nourrissons, les personnes de plus de 65 ans). ${ }^{[6]}$

Au $21^{\mathrm{e}}$ siècle, une des particularités majeures des vaccins porte sur les maladies dont ils sont destinés à diminuer le risque. Il s'agit de maladies graves (méningites, septicémies...) mettant en jeu le pronostic vital des personnes qui en sont affectées. Il s'agit aussi souvent de maladies dont les taux d'incidence restent modestes. Dès lors, l'efficacité (et les premières bases de l'argumentation de l'innocuité) doivent être démontrées au travers d'essais cliniques portant sur un nombre très important d'individus (30 000 à 70000 sujets) [figure 1]. ${ }^{[7-9]}$

Une autre particularité importante porte sur les risques d'effets indésirables des vaccins. En effet, le bénéfice collectif escompté d'un vaccin est généralement supérieur à la somme du bénéfice pour chacune des personnes vaccinées. En ce sens, décider d'accompagner la mise sur le marché d'un nouveau vaccin par un programme de vaccination engage beaucoup plus la responsabilité des décideurs de santé publique que des décideurs médicaux prescripteurs de l'acte de vaccination. Il y a de ce fait pour le vaccin encore plus que pour tous les autres médicaments une nécessité «politique » d'anticipation et de réactivité au risque de survenue d'effets indésirables; impératif renforcé par l'impact potentiel d'une gestion approximative des effets secondaires vaccinaux qui peut conduire à un échec de la politique vaccinale et de ce fait à une perte de chance au regard de la maladie infectieuse concernée même pour les personnes qui ne se seraient pas fait vacciner. Cela s'est vu par le passé. Bien sûr, tout comme pour les autres médicaments, en complément de la pharmacovigilance classique, les Plans de Gestion du Risque (Européen et Français) ${ }^{[7,10]}$ et les études post-enregistrement demandées dans le cadre de l'inscription au remboursement ${ }^{[11]}$ sont importants pour le suivi de la tolérance des vaccins et de leur intérêt de santé publique (impact en termes de morbidité, mortalité, recours aux soins, épidémiologie et écologie microbienne). 
Force est de constater qu'il n'existe pas actuellement en France d'instruments permettant d'assurer des investigations de la taille nécessaire au développement ou au suivi de certains vaccins. Ces travaux sont donc notamment réalisés aux États-Unis, où certaines organisations de gestion de santé disposent de systèmes d'information performants, comme c'est le cas de Kaiser Permanente (Health Maintenance Organization), ${ }^{[12]}$ ou du Department of Veterans Affairs (Institution publique) ${ }^{[13]}$ Certains pays Européens (Danemark, Suède, Norvège, Pays Bas....) ${ }^{[14]}$ disposent également de registres, systèmes de collection de l'information à grande échelle ou de plateformes permettant la réalisation des essais relatifs aux vaccins.

Depuis 2008, s'est mis en place en France un Réseau National d'Investigations Cliniques (REIVAC) créé à l'initiative de l'Inserm dans le cadre des Réseaux thématiques des CIC. En 2010, ce réseau regroupe une dizaine de centres d'investigations cliniques et de services cliniques et bénéficie pour la $3^{\text {ème }}$ année consécutive du soutien du Centre National de Gestion des Essais de Produits de Santé (GIP CeNGEPS). Les missions du REIVAC sont de promouvoir le développement de la recherche clinique en vaccinologie en France : recherche institutionnelle en partenariat avec l'Institut de Microbiologie et Maladies Infectieuses (IMMI) de l'Inserm, recherche industrielle en favorisant l'attractivité de la France pour la réalisation des essais vaccinaux.

\subsection{Objectif et propositions de la Table Ronde}

\subsubsection{Objectif}

Le vaccin étant potentiellement un domaine «thérapeutique» d'excellence pour la France, la Table Ronde a identifié la nécessité de pouvoir développer en France une «plateforme» permettant de réaliser des essais de phase III et investigations de phase IV. Cela nécessite l'accès à un suivi de population caractérisée à large échelle (cohortes).

\subsubsection{Propositions}

- Structuration en réseau et fédération des experts académiques en vaccinologie, composé notamment d'épidémiologistes, de viro-bactériologistes, d'immunologistes, de cliniciens, de méthodologistes et de sociologues; cette fédération pourrait venir compléter et élargir les compétences déjà regroupées au sein du REIVAC.

- Constitution d'une base de données dans le but de constituer un système d'information préexistant nécessaire aux essais de phase III, aux études observationnelles, ou aux Plans de Gestion du Risque des vaccins.
La base de données GPRD (General Practice Research Database - Royaume Uni) constitue un exemple de référence de base de données «patients » dont la France pourrait se doter. ${ }^{[15]}$

Différents organismes de gestion ou d'organisation de la Santé en France disposent de bases de données spécifiques à leur domaine d'activité. C'est le cas notamment de la Caisse Nationale d'Assurance Maladie (Cnamts), qui dispose via son système de gestion et ses bases statistiques des informations relatives aux soins de l'ensemble des bénéficiaires de l'assurance maladie. ${ }^{[16]}$ De même, l'Agence Technique de l'Information sur l'Hospitalisation gère le Programme de Médicalisation des Systèmes d'Information Hospitaliers (PMSI), système d'information qui enregistre les pathologies et les prises en charge relatives aux patients traités en établissements de soins (publics et privés) en France. ${ }^{[17]}$ Citons également les informations collectées par les 31 Centres Régionaux de Pharmacovigilance. La mise en réseau des bases de données existantes en France, et leur ouverture à but de recherche et de développement clinique comme proposé pour certaines bases par l'Institut des Données de Santé, pourrait constituer une plateforme de choix permettant le développement clinique et le suivi des nouveaux vaccins en France.

- Constitution de données partagées sur les comportements, permettant l'accès à des données du type «nombre de sujets contacts », ou de couverture vaccinale.

L'évaluation des nouvelles stratégies vaccinales nécessite de modéliser l'impact épidémiologique attendu des nouvelles vaccinations. Ces modélisations impliquent de pouvoir disposer de matrices de contact, reflétant les probabilités de contacts contaminants entre sujets. Pour être pertinentes, ces matrices doivent être adaptées aux spécificités françaises, incluant par exemple l'âge de mise en collectivité des enfants, la nature des structures d'accueil collectif des enfants en fonction de l'âge, les différents types d'établissements... Les modèles étant très sensibles au choix des matrices de contacts, ${ }^{[18]}$ la constitution de données partagées sur les comportements (nombre de sujets contacts, couverture vaccinale) est un préalable à une évaluation de qualité des nouvelles vaccinations.

- Création d'une Très Grande Infrastructure de Recherche épidémiologique (TGIR).

Les TGIRs sont des outils établis en vue de mener des recherches d'importance et pouvant assurer une mission de service pour une ou plusieurs communautés scientifiques de grande taille. Les TGIRs sont très différentes selon leur finalité, leur budget, ou la taille de leur communauté d'utilisateurs. Le domaine de la biologie et de la santé font l'objet de TGIR, existantes, décidées ou en projet (Réseau des Centres d'Investigation Clinique, Plate-forme de Recherche et Développement des grandes Cohortes Biomédicales...). ${ }^{[19]}$ 


\title{
Continuum soins-recherche : du patient au patient
}

\author{
Faire bénéficier le plus vite possible les patients
} des découvertes les plus récentes

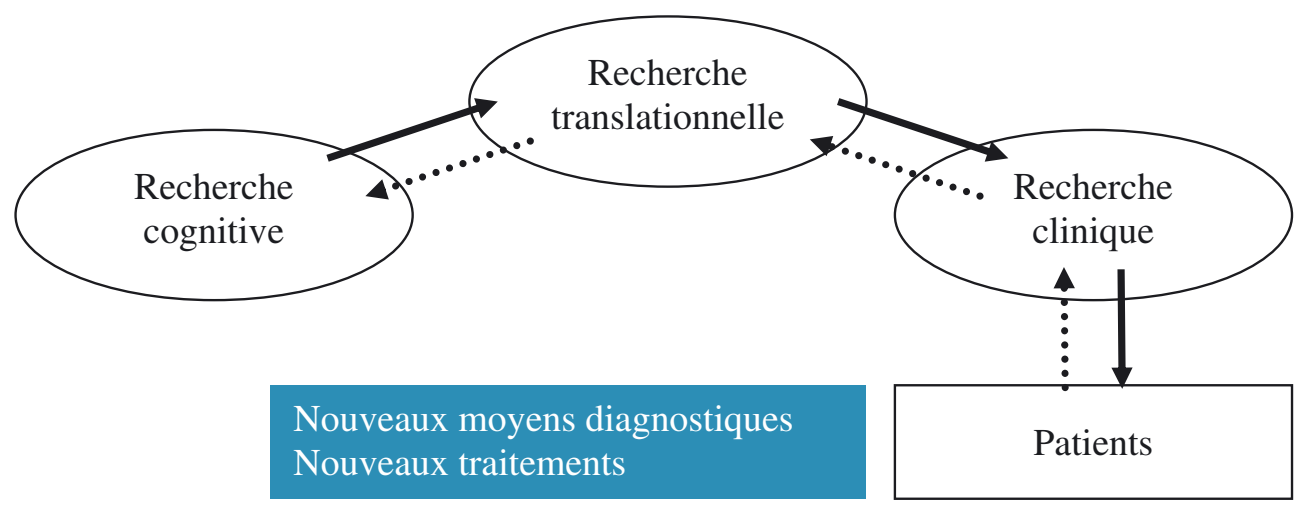

Fig. 2. La recherche translationnelle : du malade au malade. ${ }^{[20]}$

L'épidémiologie permet d'observer les populations, les maladies, de générer des hypothèses sur les déterminants de l'état de santé et l'évolution des infections et pathologies. Elle constitue donc le socle de la recherche fondamentale de tout nouveau vaccin. Elle est aussi un élément d'éclairage indispensable aux prises de décisions en matière de vaccination. Dès lors, au regard de l'importance de la recherche épidémiologique, et de la multiplicité des communautés scientifiques intéressées, une TGIR en recherche épidémiologique aurait tout son sens.

- Développement de centres de recherche translationnelle, de façon à concurrencer les structures disponibles dans les autres pays et assurer la visibilité de la France en matière de développement des vaccins.

La recherche translationnelle a pour objectif d'assurer le continuum recherche-soins et l'application la plus rapide possible des découvertes au bénéfice des patients. L'étape translationnelle, qui part de l'identification de cibles ou de produits candidats pour aboutir à la preuve du concept clinique, correspond à un éventail de savoir-faire et nécessite une infrastructure professionnalisée fournissant un ensemble de services aux communautés de chercheurs (figure 2). ${ }^{[20]}$

La recherche translationnelle, également appelée médecine expérimentale, a été crée il y a plus de 20 ans aux États-Unis.

La création par l'Inserm des Centres d'Investigation Cliniques (CIC) a pour objectif de faciliter la mise en place de projets de recherche transationnelle. Il existe actuellement un CIC dédié à la vaccinologie, le CIC BT505 localisé à l'hôpital Cochin à Paris, qui fait l'objet d'un partenariat avec l'Institut Pasteur.

En France, le Plan Cancer 2003-2007 a mis la Recherche Translationnelle au cœur de son programme de recherche, sous la coordination des cancéropoles, avec de nombreux partenariats public/privé. La recherche translationnelle est également en cours d'application à d'autres domaines (maladie d'Alzheimer, projet de TGIR en matière de recherche translationnelle dans le domaine des maladies neurologiques/maladies rares).

Le développement de nouveaux Centres de recherche translationnelle spécialisés dans la prévention des maladies infectieuses constituerait sans nul doute un environnement favorable à l'arrivée de nouveaux vaccins développés en France.

\section{Spécificités en termes d'utilisation des modèles dans la définition des nouvelles stratégies vaccinales}

\section{1. État des lieux}

L'approche et la définition des nouvelles stratégies vaccinales se basent notamment sur la modélisation des impacts épidémiologiques et économiques attendus de l'introduction de la vaccination. ${ }^{[21]}$ Cette approche est menée sous l'égide du Comité technique des vaccinations, et implique différents partenaires, dont l'Institut de Veille Sanitaire (InVS). ${ }^{[22]}$

Les modèles développés peuvent être très complexes (de statiques à dynamiques), et prennent en considération :

- L'histoire naturelle de la maladie visée, l'épidémiologie de l'infection, la prise en charge actuelle de la maladie, ainsi que les moyens de prévention existants.

- L’impact attendu du vaccin (bénéfices, risques) sur les agents infectieux et leur évolution, pour les vaccinés (bénéfice individuel), pour les non vaccinés (impact collectif lié à l'immunité populationnelle), pour l'assurance maladie, pour la société (productivité, arrêts de travail évités). 
Dans ce contexte, la mise à disposition de bases de données sur les diagnostics et soins, ainsi que sur les comportements telles que décrites précédemment - permettrait d'avoir accès à des données épidémiologiques et économiques françaises de qualité afin de «nourrir » les modèles avec les données les plus pertinentes.

La complexité inhérente aux modèles à développer pour évaluer l'impact attendu du vaccin, et pour collecter certaines des données nécessaires aux analyses, peut avoir pour conséquence de générer des délais dans les avis du Comité Technique des Vaccinations (CTV).

La Procédure de traitement des dossiers vaccins par le Comité Technique des Vaccinations (février 2010) précise d'ailleurs : «Le projet d'avis du CTV est rendu dans la mesure du possible dans un délai de 3 mois à compter de l'avis positif de l'Agence Européenne du Médicament ou de la fin de la procédure de reconnaissance mutuelle ou de la procédure nationale. Lorsque des données complémentaires ont été demandées au laboratoire, ou lorsque des études complémentaires sont nécessaires, notamment une modélisation mathématique ou une évaluation médico-économique, ce délai pourra être prolongé en fonction du temps nécessaire à la mobilisation des ressources et de la complexité des travaux ».

\subsection{Proposition}

La Table Ronde a identifié :

- Le besoin d'anticiper au plus tôt la préparation du/des modèle(s) et la collecte des données nécessaire à l'évaluation des nouvelles stratégies vaccinales, en parallèle de l'évaluation réglementaire Européenne, afin que les délais d'accès de la population aux nouveaux vaccins ne soient pas accrus outremesure par la modélisation.

- Le besoin de formaliser les interactions entre agences, opérateurs de recherche et industriels dans le développement des modèles.

\section{Spécificités en termes d'évaluation et d'accès au remboursement des nouveaux vaccins}

\section{1. État des lieux}

Le parcours des vaccins pour obtenir le remboursement est marqué par la double nature du vaccin, qui est à la fois un médicament et un outil de santé publique. Le vaccin est donc soumis à un processus réglementaire distinct.

\subsubsection{Un parcours marqué par une étape supplémentaire}

En tant que médicament, le nouveau vaccin fait classiquement l'objet d'un avis de la Commission de la Transparence de la Haute Autorité en Santé (HAS), qui évalue le Service Médical Rendu (SMR) et l'Amélioration du Service Médical Rendu (ASMR), et d'une décision du Comité Économique des Produits de Santé (CEPS) qui en fixe le prix.

Mais sa qualité concomitante d'instrument de santé publique lui vaut en plus, d'être évalué par une troisième instance, le CTV. C'est un comité technique permanent composé d'experts des questions vaccinales et rattaché au Haut Conseil de la Santé Publique (HCSP), l'organe consultatif placé auprès du Ministre de la Santé pour contribuer à la définition, l'évaluation et la réflexion sur la politique de santé publique en France. In fine, la politique de vaccination est élaborée directement par le ministre de la santé, ${ }^{[23]}$ qui fixe les conditions d'immunisation, énonce les recommandations nécessaires et rend public le calendrier des vaccinations après avis du HCSP.

Le CTV a pour missions :

- D'assurer la veille scientifique sur les évolutions et les perspectives en matière de vaccins.

- D'élaborer la stratégie vaccinale en fonction des données épidémiologiques, des études bénéfice-risque individuel et collectif et des études médico-économiques relatives aux mesures envisagées.

- De proposer des adaptations en matière de recommandations et d'obligations vaccinales pour la mise à jour du calendrier vaccinal (figure 3 ).

\subsubsection{Un parcours marqué par une double évaluation économique}

En France, l'évaluation médico-économique des médicaments par la HAS est principalement réalisée post-inscription au remboursement, lors de la réévaluation des classes thérapeutiques, ou lors de l'évaluation des stratégies de prise en charge. ${ }^{\text {[24] }}$

En ce qui concerne les vaccins, les participants de la Table Ronde se sont accordés sur le fait que ces produits sont une exception à la règle d'absence d'évaluation médico-économique en première inscription appliquée aux autres médicaments, vue l'importance de ce sujet dans la détermination de la stratégie vaccinale.

La dimension médico-économique doit donc faire partie de l'évaluation menée par le CTV préalablement au remboursement, lors de l'étape de recommandation. Ensuite, les questions économiques sont de nouveau abordées par le CEPS, lors de la fixation du prix. 


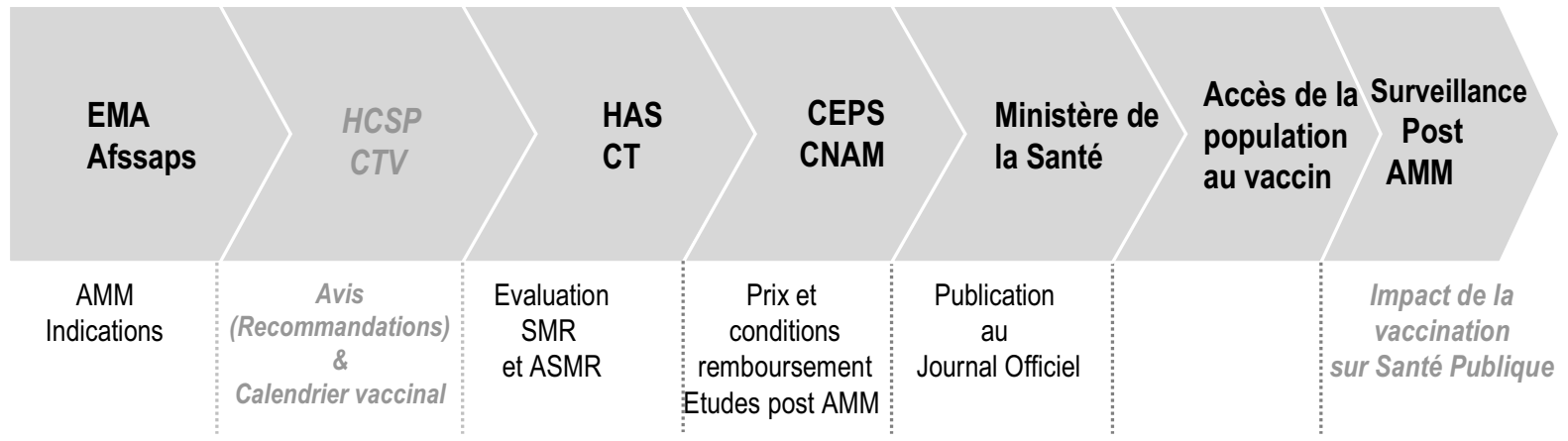

En italique, étape supplémentaire spécifique aux vaccins Les autres étapes sont communes pour tous les produits de santé (incluant vaccins \& médicaments)

EMA : European Medicines Agency; Afssaps : Agence Française de Sécurité Sanitaire des Produits de Santé ; CEPS : Comité Economique des Produits de Santé CNAM : Caisse National d'Assurance Maladie ; CTV : Comité Technique des Vaccinations ; HAS : Haute Autorité de Santé ; HCSP : Haut Conseil de Santé Publique ; (A)SMR : (Amélioration du) Service Médical Rendu

Fig. 3. Vaccins : le parcours du remboursement.

Il en résulte que deux acteurs - le CTV et le CEPS - réalisent une évaluation économique impliquant le même vaccin, et que cet état de fait pose la question de leur coordination, notamment sur la question du prix du vaccin, dans le cas où le CTV conduit des analyses médico-économique sur la base d'hypothèses de prix ou de conditions de remboursement qui n'ont pas encore été évaluées par le CEPS.

Il est apparu au cours des discussions de la Table Ronde :

- Un manque de clarté sur les rôles de chacun des acteurs, un déficit de communication et de coordination.

- La nécessité de préciser où et quand est faite l'évaluation médico-économique, et pour quel(s) décideur(s).

\subsection{Objectif et propositions}

\subsubsection{Objectif}

Anticiper et coordonner l'évaluation des nouveaux vaccins en France.

\subsubsection{Propositions}

- Mise en place d'un réseau identifié d'experts «Évaluation des stratégies vaccinales », intégrant notamment des vaccinologues, des économistes et des modélisateurs.

- Développement d'une interface renforcée entre les instances évaluant les stratégies vaccinales et les vaccins (Afssaps/CTV/ HCSP/HAS/CEPS), avec notamment :
- un accord par l'ensemble des instances des missions respectives en matière de vaccin et de vaccination,

- la clarification du processus complet menant le vaccin de l'autorisation de mise sur le marché jusqu'au remboursement,

- la création d'un Comité de liaison temporaire entre les différentes instances impliquées dans l'évaluation du vaccin.

- Amélioration de l'interface entre les instances évaluant les stratégies vaccinales/vaccins et les industriels, avec la définition et la communication de procédures de fonctionnement consensuelles.

À ce titre, la Procédure de traitement des dossiers vaccins par le CTV (Février 2010) constitue une première étape dans ce processus.

\section{Spécificités des vaccins en termes de perception et de communication}

\section{1. État des lieux}

Les vaccins sont des produits de santé totalement à part :

- Aucun progrès technologique (à part l'eau potable) - y compris les antibiotiques- n'a eu autant d'influence que la vaccination sur la réduction de la mortalité et la croissance de la population mondiale, ${ }^{[25]}$

- Au sein de la thérapeutique, il s'agit probablement de celle qui a fait l'objet du plus de controverses, et cela continue (postérieurement à la table ronde, la période de pandémie grippale AH1N1 en a fourni une éclatante démonstration). 
Opinions favorables («très favorables » et«plutôt favorables ») à la vaccination en général, selon le sexe, parmi les 15 à 75 ans

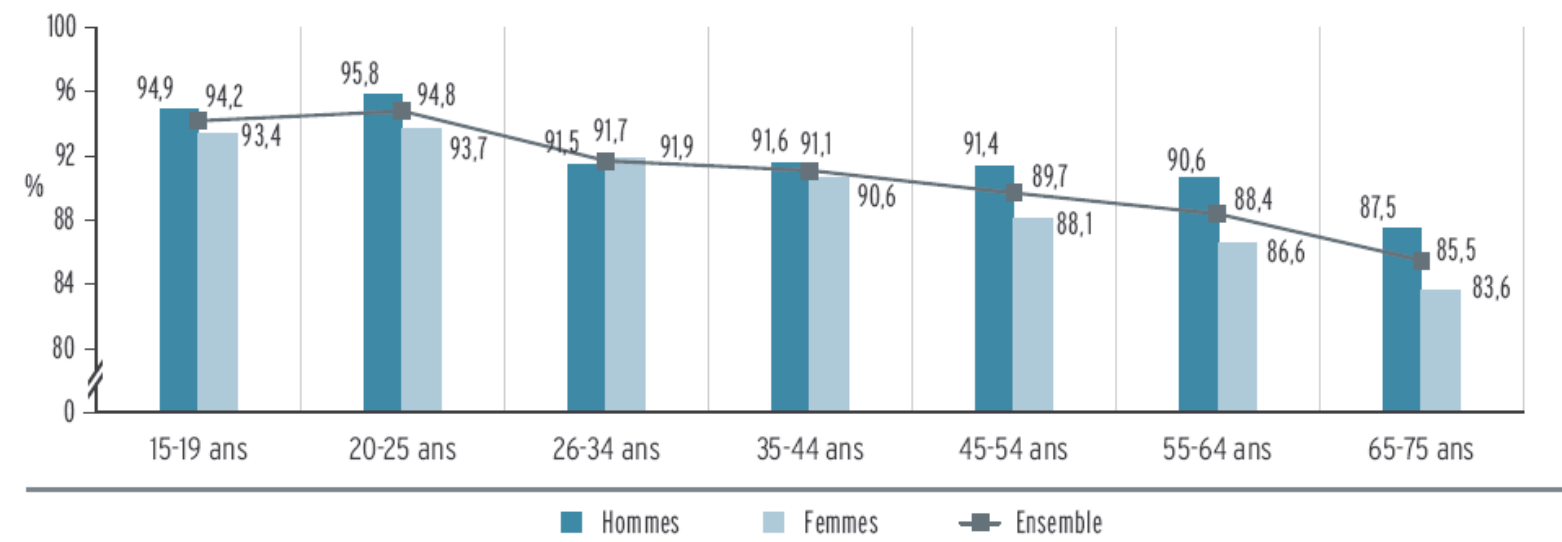

Fig. 4. Opinions du grand public vis-à-vis des vaccinations. ${ }^{[27]}$

Perception déclarée de la vaccination par la population et les médecins généralistes : les enquêtes d'opinion montrent qu'environ $90 \%$ de la population est favorable à la vaccination, et qu'il y a moins de $5 \%$ d'opposants farouches.

De même, $97 \%$ des généralistes se déclarent favorables à la vaccination, dont $75 \%$ très favorables. ${ }^{[26]}$ Il est à noter que les médecins traitants réalisent $90 \%$ des vaccinations en France, d'où un rôle majeur dans l'information et la perception de la population vis-à-vis des vaccins (figure 4).

Réalité de la vaccination : les couvertures vaccinales en France ne sont pas à la hauteur de ces chiffres optimistes. Si la couverture vaccinale est satisfaisante pour les vaccinations du nourrisson (98\% à 2 ans pour les pentavalents), elle se dégrade avec l'âge (80 \% pour la vaccination Diphtérie-TétanosPoliomyélite en classe de $3^{\text {ème }}$ ), et devient problématique chez l'adulte (après 65 ans : environ $60 \%$ de couverture pour Tétanos, $10 \%$ pour Diphtérie). Cette insuffisance de couverture vaccinale s'est traduite récemment par le développement de foyers épidémiques de rougeole. À noter un taux de couverture extrêmement bas pour la vaccination Hépatite B en France, résultant des controverses autour de sa sécurité.

Cette réalité traduit l'existence dans une partie de la population et de la communauté médicale d'un scepticisme vis-à-vis de la vaccination, voire d'une opposition.

Sceptiques et opposants : les sceptiques à la vaccination ne rejettent pas l'ensemble des vaccinations, mais ont plutôt une démarche sélective. Ils sont également très demandeurs d'informations sur la stratégie vaccinale, son efficacité et sa sécurité.

Les opposants à la vaccination (estimés à moins de $5 \%$ de la population) constituent un groupe hétérogène animé par des motivations très diverses : croyances religieuses, philosophiques ou idéologiques; revendication du respect des libertés individuelles; attirance vers des médecines alternatives; croyance en une «Théorie du complot».

Il est à noter que dans cette opposition plus ou moins marquée, le rôle des médecins est probablement très important, car ils constituent pour la population les référents en matière de vaccination.

De façon générale, on note un besoin croissant d'informations et d'explications autour de la vaccination (gravité de la maladie prévenue, efficacité et innocuité du vaccin) [tableau I].

Nécessité d'améliorer la communication autour de la vaccination : de façon générale, notre époque est marquée par une demande d'information accrue sur tous les sujets : la santé, et en particulier la vaccination, n'échappe pas à cette évolution. Plus que tout autre domaine de santé, pour être acceptées, les vaccinations doivent être expliquées et comprises.

Cette demande émane de la population, mais aussi des médecins traitants, qui sont les principaux acteurs de la vaccination en France.

\subsection{Objectifs et propositions de la Table Ronde}

- Informer le public et les professionnels de santé :

- Accroître la médiatisation de la Semaine Européenne de la Vaccination.

- Communiquer les conclusions des recommandations vers le public et les professionnels, avec par exemple l'organisation de conférences de presse du CTV/HCSP lors de l'émission de recommandations importantes.

- Développement d'un site internet officiel sur la vaccination destiné au grand public. 
Tableau I. Arguments « anti-vaccination » : quelles leçons en tirer? Quelles mesures à prendre? Quelle réponse apporter?

\begin{tabular}{ll}
\hline Argument « anti vaccination » ${ }^{[2,29]}$ & Mesure à prendre ou réponse à apporter \\
\hline $\begin{array}{l}\text { Vacciner = une violation des droits de l'in- } \\
\text { dividu }\end{array}$ & $\begin{array}{l}\text { S'interroger sur les obligations vaccinales : plus on impose de mesures coercitives, } \\
\text { plus on renforce les opposants à la vaccination }\end{array}$ \\
Vacciner = le fruit d'un complot & Nécessité d'une réflexion sur la gestion de certains liens entre les experts en vaccino- \\
& logie et l'industrie (y compris les participations aux congrès) \\
Vacciner = danger & Anticiper d'éventuelles «relations temporelles » lors de la mise en place des nouvelles \\
& vaccinations, avec une communication adaptée en direction des médecins. \\
& Démontrer que le système de pharmacovigilance des vaccins est sérieux, et probable- \\
& ment l'améliorer \\
& Analyser les événements indésirables notifiés (notamment dans les essais cliniques) \\
& en matière d'imputabilité \\
& Répondre aux questions que se posent les médecins sur la vaccination (exemple : In- \\
fovac), et les aider à répondre à la population. Pour chaque nouvelle recommandation, \\
produire une version simplifiée adressée pour publication aux journaux que lisent les \\
médecins
\end{tabular}

- Communication annuelle par l'Afssaps des données des Plans de Gestion du Risque (comme cela est déjà le cas pour les vaccins contre les infections à HPV), destinée au public et aux professionnels de santé.

- Former les professionnels de santé

- «Institutionnaliser» le financement d'Infovac. ${ }^{\text {[30] }}$

- Accroître la formation des professionnels de santé à la prévention et à la vaccination.

- Impliquer

- Inviter en tant qu'observateurs des représentants de la société civile aux réunions du CTV (à l'instar du fonctionnement de l'organisme américain en charge des recommandations vaccinales).

- Proposer des débats citoyens sur la vaccination (ex. de thème : l'obligation vaccinale).

- Réfléchir à la place des industriels/académiques.

Un autre point abordé par la Table Ronde a été celui de la gestion des conflits d'intérêt des experts en vaccinologie : ce sujet pourra faire l'objet d'une table ronde en 2010.

\section{Conclusion}

Les Rencontres Nationales de Pharmacologie Clinique de Giens XXV ont donné l'opportunité à la Table Ronde $\mathrm{n}^{\circ} 6$ de dresser un état des lieux des spécificités du vaccin, et de formuler une liste de propositions ambitieuses.

L'expérience récente de la vaccination contre la grippe pandémique H1N1 en France a montré que le développement de vaccins efficaces et sûrs, la coordination entre les acteurs de santé publique et la communication autour des stratégies vaccinales constituent de vrais enjeux de santé publique. Les propositions formulées par la Table Ronde visent à améliorer les processus dans ces grands domaines spécifiques au vaccin.

La Table Ronde a proposé la création d'un groupe de travail, qui devra s'assurer de la poursuite, du suivi et de la mise en œuvre des propositions formulées.

Participants. Véronique Ameye (Laboratoire Novartis Pharma SAS), Nathalie Billon (Laboratoire Sanofi Aventis), Pierre-Yves Boelle (Université Pierre et Marie Curie, Paris), Jocelyn Courtois (Cnamts, Paris), Benoît Dervaux (DRCI, CHRU, Lille), Isabelle Diaz (Leem-Ariis, Paris), Jean-Paul Fagot (Afssaps, Paris), Daniel Floret (Hôpital Femme Mère Enfant, Bron), Bernard Fritzell (Laboratoire Wyeth Pharmaceuticals), Anne-Françoise Gaudin (Laboratoire GlaxoSmithKline), Gaëlle Gernigon (DGS, Paris) Chrystel Jouan-Flahault (Leem-Ariis, Paris), Catherine Lassale (Leem-Ariis, Paris), Hervé Le Louet (CRPV, Hôpital Henri Mondor, Paris), Daniel Levy-Bruhl (INVS, Saint Maurice), François Meyer (HAS, Saint-Denis), Sophie Muller (Laboratoire GlaxoSmithKline), Benoît Soubeyrand (Laboratoire Sanofi Pasteur MSD), Bernard Teisseire (CEPS, Ministère de la Santé, Paris).

\section{Références}

1. Ministère de la Santé et des Solidarités. Avis du comité technique des vaccinations et du conseil supérieur d'hygiène publique de France relatif à la vaccination contre les papillomavirus humains $6,11,16,18.9$ mars 2007 http://www. sante-sports.gouv. fr/dossiers/cshpf/a mt_090307_papillomavirus.pdf

2. Haut Conseil de la Santé Publique. Avis relatif à la vaccination contre les papillomavirus humains 16 et 18 par un vaccin bivalent. 14 décembre 2007 http://www.hcsp.fr/docspdf/avisrapports/ hcspa20071214_Papillomavirus.pdf

3. Ministère de la Santé et des Solidarités. Avis du conseil supérieur d'hygiène publique de France relatif à la vaccination par le vaccin antipneumococcique conjugué chez les enfants de moins de deux ans et les 
enfants de deux à cinq ans. 19 mai 2006 http://www. sante.gouv.fr/ htm/dossiers/cshpf/a_mt_190506_pneumo_enf.pdf

4. Haut Conseil de la Santé Publique. Avis relatif à la vaccination par le vaccin méningoccique conjugué de sérogroupe conjugué. 24 avril et 26 juin 2009 http://www.hcsp.fr/explore.cgi/avisrapportsdomaine? ae=avisrapportsdomaine $\&$ clefdomaine $=1 \&$ menu $=09$

5. Leem. 50 questions sur les vaccins. http://www.leem-media.com/ leem/Communiques/Dossiers-de-presse/Innovation-RechercheProgres-therapeutique-Vaccins/Les-vaccins-bouclier-de-1avenir

6. Ministère de la Santé et des Sports. Calendrier vaccinal détaillé 2009. http://www . sante-sports.gouv. fr/calendrier-vaccinaldetaille-2009.html

7. EMEA European Public Assessment Reports Zostavax ${ }^{\circledR}$ http://www.ema . europa.eu/humandocs/Humans/EPAR/zostavax/zostavax.htm

8. EMEA European Public Assessment Reports Rotateq ${ }^{\circledR}$. http://www . ema. europa. eu/humandocs/Humans/EPAR/rotateq/rotateq.htm

9. EMEA European Public Assessment Reports Prevenar ${ }^{\circledR}$. http://www . ema . europa.eu/humandocs/Humans/EPAR/prevenar/prevenar.htm

10. Afssaps. Plans de gestion des risques Gardasil et Cervarix ${ }^{\circledR}$. http: //www . afssaps.fr/Activites/Plans-de-gestion-des-risques

11. Haute Autorité de Santé. Avis de la Commission de la Transparence Gardasil du 18 avril 2007. http://www.has-sante.fr/portail/jcms/ c_523363/gardasil

12. Kaiser Permanente. http://xnet.kp.org/clinicaltrials/

13. United States Department of Veterans Affairs. http://www.va.gov

14. Julius Center. Clinical Trial Monitoring. http://www.juliuscentrum. nl/julius/Services/ClinicalTrialMonitoring/tabid/861/ Default.aspx

15. General Practices Research Database (GPRD). http://www . gprd.com

16. Assurance Maladie. Données statistiques. http://www.ameli.fr/lassurance-maladie/statistiques-et-publications/donneesstatistiques/index.php

17. ATIH. http://www .atih. sante. fr

18. Bonmarin I, Santa-Olalla P, Bernillon P, et al. Modélisation de l'impact de la vaccination sur l'épidémiologie de la varicelle et du zona. Août 2008. http://www.invs.sante.fr/recherche/index2.asp? txtQuery=varicelle+zona

19. Roadmap française des très grandes infrastructures de recherche (TGIR). 9 décembre 2008 http: //www . roadmaptgi.fr/
20. ARIIS. Symposium Leem Recherche INCa sur la recherche translationnelle en cancérologie. http://www.leem-recherche.org/ 8/335-symposium-leem-recherche-inca-sur-la-recherchetranslationnelle-en-cancerologie-presentations-en-ligne/

21. Ministère de la Santé, de la Jeunesse et des Sports. Arrêté du 18 septembre 2007 relatif au Comité technique des vaccinations. http://textes . droit.org/JORF/2007/09/27/0224/0027/

22. InVS. La modélisation mathématique : un outil d'aide à la décision en situation d'incertitude. Novembre 2008. http://www . invs . sante. fr/recherche/index2 . asp? txtQuery=mod\%E9lisation+math\%E9matique

23. République française. Article L. 3111-1 de la Loi du 4 août 2004 du Code de la Santé Publique. http://www.legifrance.gouv.fr/html/actualite/ actualite_legislative/decrets_application/2004-806.htm

24. Haute Autorité de Santé (HAS). Nouvelle organisation des Commissions de la HAS. Communiqué de presse. 4 juillet 2008. http://www.hassante.fr/portail/jcms/c_676358/nouvelle-organisation-descommissions-de-la-has

25. Plotkin SL, Plotkin SA. Chapter one. In: Plotkin and Orenstein. Vaccines 2008

26. INPES. Baromètre pharmaciens/medecins 2003. http://www . inpes . sante. fr/index . asp?page=CFESBases/catalogue/ detailspecial . asp?numfiche=793\&order=undefined

27. INPES. Baromètre santé 2005 http://www.inpes.sante.fr/ index . asp?page=CFESBases $/$ catalogue/detailspecial . asp? numfiche $=1109 \backslash$ \&order=undefined

28. Davies P, Chapman S, Leask J. Antivaccination activists on the world wide web. Arch Dis Child 2002 Jul; 87(1): 22-5

29. Wolfe RM. Vaccine safety activists on the Internet. Expert Rev Vaccines 2002 Oct; 1(3): 249-52

30. http://www.infovac. fr

Correspondance et offprints : Odile Launay, Hôpital Cochin, CIC de Vaccinologie Cochin Pasteur, 27 rue du Faubourg Saint-Jacques, 75679 Paris Cedex 14, France.

E-mail : odile.launay@cch.aphp.fr 